\title{
Spin-Orbit Interaction in a Spherical Quantum Dot
}

\author{
I.F.I. Mikhail, I.M.M. Ismail and M.M. El Shafee \\ Department of Mathematics, Faculty of Science, Ain Shams University, Cairo, Egypt \\ (Received May 30, 2013; in final form December 30, 2013)
}

\begin{abstract}
The conventional spin-orbit interaction due to the presence of an off-center impurity located in a spherical quantum dot of finite confining potential has been investigated. The different effective masses of dot and barrier are taken into consideration. The spin-orbit interaction has been calculated in the excited state $(2 p)$. The variational method has been applied by using a new form of the trial wave function in addition to the conventional form that has been used in previous work. The new form has the advantage of satisfying the boundary conditions at the interface between dot and barrier in the case of different masses. It has been shown that the spin-orbit interaction takes its highest value when the impurity is located in the vicinity of the position at which the radial electron probability takes its maximum value. The corresponding results of a central impurity has been investigated as the limiting case when the impurity radial coordinate tends to zero. The case of central impurity has been further explored by using the exact solution in the state $(2 p)$ of the radial Schrödinger equation in the presence of the impurity.
\end{abstract}

DOI: $10.12693 /$ APhysPolA.125.1197

PACS: 71.55.-i, 71.70.Ej, 73.21.La, 75.70.Tj

\section{Introduction}

Recently, there has been a great interest in studying spin-orbit interactions in low dimensional structures due to their important role in spin transport and in spintronic devices. The previous studies are concerned with three types of spin-orbit interactions namely, Rashba [1-18], Dresselhaus $[4,11,12,14,16,19,20]$ and conventional interactions [21-25]. The study of Rashba and Dresselhaus interactions have been mainly considered in two-dimensional geometries. Thus they cannot be investigated in a spherical quantum dot which is an isotropic geometry unless if a magnetic or an electric field is applied. It seems, therefore, that the conventional interactions present the most important type of spin-orbit interaction in quantum dots. In our opinion very little has been done to explore this type of interactions and further investigations are still needed. Besides, it has been pointed out in Bella and Navaneethakrishnan [22] that the Rashba interactions are negligible with respect to the conventional interactions in GaAs based structures.

The present work is concerned with studying the spinorbit interaction in a quantum dot. The interaction arises due to the presence of a center or off-center impurity located in the dot. The different effective masses of dot and barrier are taken into consideration. The electron energy and binding energy have to be calculated. For a central impurity, the binding energy without taking the spinorbit interaction into consideration has been mainly investigated by using the variational method and the exact analytical approach. For the off-center impurity no exact solution has been obtained and accordingly the variational method can only be employed. Regarding the exact solution, the series solution method was used by Zhu and his co-workers [26-29]. The convergence of the series solution was found to be slow at some points. Zhu et al. $[27,28]$ were forced to use uniformly convergent Taylor expansions around these points. This indicates that the solution obtained in these cases may deviate to a certain extent from the exact solution. Along the same direction Chuu et al. [30], Yang et al. [21] and Goldman and Joslin [31] have derived alternative exact analytical solutions. In the last reference the simple case of infinite confining potential has been considered while in the first two references the exact solution takes different forms in the two cases when the electron energy is negative or positive. This gives rise to some problems since the electron energy is always determined from the boundary conditions that depend on the form of the wave functions. Thus, in some cases it is not clear which form of the exact solution has to be taken. Recently, Mikhail and El Sayed [32] introduced new analytical forms for the exact solution that are valid for any electron energy positive or negative. They are also valid for different masses, different dielectric constants, and finite confining potentials. The method was applied in Ref. [32] for the ground state (1s).

The variational method has been applied for both central and off-central impurities. The conventional form of the trial wave function has been used in most of the earlier works (Montenegro and Merchancano [33], Peter [34], Dane et al. [35, 36], Akbas et al. [37] and Mikhail and Ismail $[38,39])$. In Mikhail and El Sayed [32] a new form of the trial wavefunction has been introduced for the ground state $(1 s)$ for central and off-central impurities. It has the advantage that it satisfies the required boundary conditions in the case of different masses unlike the conventional trial form. Also, in Çakir et al. [40], Özmen et al. [41] and Yakar et al. [42, 43] a modified variational optimization approach in the case of central impurity has been explored. However, their approach cannot be applied to the case of off-central impurity as the elements of the basis used depend on the spherical harmonic functions.

The conventional spin-orbit interaction vanishes for the ground state $(1 s)$. Thus in order to calculate such interaction we have to consider the excited state $(2 p)$. In all previous works (Refs. [21-25]) only the case of cen- 
tral impurity has been considered by using equal effective masses of dot and barrier. In the present work we deal with both central and off-central impurities when the effect of the different masses of dot and barrier are taken into account. The off-central impurity has been dealt with by using the variational method. Two forms of the trial wavefunction have been utilized, the conventional form and a new form that has the advantage of satisfying the required boundary conditions in the case of different masses. The new form has been constructed for the state $(2 p)$ in a similar manner to the new forms introduced for the ground state $(1 s)$ in Mikhail and El Sayed [44, 45, 32] for the cases of quantum well wire (QWW), coaxial QWW and multilayered quantum dot (QD), respectively. Also, we have shown that the impurity location at which the spin-orbit interaction takes its highest value is consistent with the position at which the probability of finding the electron in the quantum dot is maximum.

A similar study was performed in Mikhail and Ismail [39] regarding the binding energy $E_{\mathrm{b}}$ of the state $(2 p)$ where it was shown that $E_{\mathrm{b}}$ takes its maximum value when the impurity is located at the position of the maximum radial electron probability. The corresponding results in the case of equal masses can be inferred by equating the masses of dot and barrier in the expressions obtained. Also, the results in the case of central impurity can be deduced by taking the limit when $r_{0} \rightarrow 0$, where $r_{0}$ is the impurity location measured from the center of the dot. The case of central impurity has further been investigated in the required excited state $(2 p)$ by employing the exact solution obtained in Mikhail and El Sayed [32]. The results obtained by using the exact solution for central impurity are higher by about $1.36 \%$ and by about $14.6 \%$ than the results obtained by using the new and conventional forms of the trial variational wave function, respectively, at $R=0.64 a^{*}$. It is thus clear that the new form of the trial wave function yields results for the spin-orbit interaction which are very close to the corresponding results of the exact approach for dots of small radius.

The paper is organized as follows. In Sect. 2, the basic equations are given in addition to the solution in the absence of impurity. The case of off-central impurity is considered in Sect. 3 by applying the variational method to calculate the electron energy, the binding energy, and spin-orbit interaction. The conventional form of the trial wave function is used in Sect. 3.1 while the new form has been introduced and applied in Sect. 3.2. Section 4 is devoted to consider the exact analytical solution for the central impurity in the state $(2 p)$. The corresponding expression for the spin-orbit interaction has been derived. Finally, the numerical results obtained in the case of $\mathrm{GaAs}_{-} \mathrm{Ga}_{1-x} \mathrm{Al}_{x} \mathrm{As}$ dots are displayed and discussed in Sect. 5.

\section{Basic equations}

The Hamiltonian operator that describes the problem of a hydrogenic donor impurity located in a spherical quantum dot at the position $r_{0}$ in the presence of the spin-orbit interaction is given by

$$
\hat{H}=\hat{H}_{1}+\frac{\hat{B}}{\epsilon\left|\boldsymbol{r}-\boldsymbol{r}_{0}\right|^{3}},
$$

where

$$
\hat{H}_{1}=\hat{H}_{0}-\frac{e^{2}}{\epsilon\left|\boldsymbol{r}-\boldsymbol{r}_{0}\right|}
$$

and

$$
\hat{H}_{0}=\frac{\hat{P}^{2}}{2 m^{*}(r)}+V(r)
$$

In Eq. (1c) $\hat{H}_{0}$ is the Hamiltonian in the absence of the impurity and spin-orbit interaction while the second terms in Eqs. (1b), (1a) represent, respectively, the Coulomb interaction between the electron and the impurity and the spin-orbit interaction. Here $\epsilon$ is defined as

$$
\epsilon=4 \pi \epsilon_{\mathrm{r}} \epsilon_{0},
$$

where $\epsilon_{\mathrm{r}}$ and $\epsilon_{0}$ are the relative and free space permittivities. Also, $m^{*}(r)$ is the effective mass of the electron and $V(r)$ is the finite potential energy of the barrier. The different effective masses of the dot and the barrier are taken into consideration, accordingly

$$
m^{*}(r), V(r)= \begin{cases}m_{1}, 0 & \text { if } 0<r<R, \\ m_{2}, V_{0} & \text { if } R<r<\infty .\end{cases}
$$

$m_{1}$ and $m_{2}$ are the effective masses of GaAs and $\mathrm{Ga}_{1-x} \mathrm{Al}_{x} \mathrm{As}$, where $m_{2}$ depends on the Al concentration $(x)$.

The spin-orbit interaction operator $\hat{B}$ is given according to Griffiths [46] by

$$
\hat{B}=\frac{e^{2}}{2 m^{* 2}(r) c^{2}} \hat{\boldsymbol{S}} \cdot \hat{\boldsymbol{L}}=\frac{e^{2}}{4 m^{* 2}(r) c^{2}}\left(\hat{J}^{2}-\hat{L}^{2}-\hat{S}^{2}\right),
$$

where $c$ is the speed of light, $\hat{L}, \hat{S}$ and $\hat{J}$ are the angular momentum, spin and total angular momentum operators. It is worthwhile noticing that for the hydrogenic states $(1 s)$ and $(2 s)$ the operator $\hat{B}$ and accordingly the spinorbit interaction vanish, since $\hat{L}=0$ (the orbital angular momentum quantum number $l=0$ ). For this reason, we consider in the present work the hydrogenic excited state $(2 p)$ for which $l=1$.

In the rest of the article the reduced units of the Bohr radius $a^{*}=\hbar^{2} \epsilon /\left(m_{1} e^{2}\right)$ and effective Rydberg $R^{*}=m_{1} e^{4} / 2 \hbar^{2} \epsilon^{2}$ will be used, for simplicity.

Now, in the absence of the hydrogenic impurity and the spin-orbit interaction, the exact electron eigenfunction of the Schrödinger equation corresponding to the Hamiltonian $\hat{H}_{0}$ in the ground state is given by (Ref. [22])

$$
\phi(r)= \begin{cases}\phi_{1}=N \frac{\sin \left(k_{1} r\right)}{r}, & 0<r<R, \\ \phi_{2}=N \frac{\sin \left(k_{1} R\right)}{r} \mathrm{e}^{k_{2} R} \mathrm{e}^{-k_{2} r}, & R<r<\infty .\end{cases}
$$

The corresponding eigenvalue $E_{0}$ is given in Rydberg units by

$$
E_{0}=k_{1}^{2}=V_{0}-\frac{m_{1}}{m_{2}} k_{2}^{2} .
$$

Also, the boundary conditions that have to be satisfied are 


$$
\phi_{1}(R)=\phi_{2}(R),\left.\quad \frac{1}{m_{1}} \frac{\partial \phi_{1}}{\partial r}\right|_{r=R}=\left.\frac{1}{m_{2}} \frac{\partial \phi_{2}}{\partial r}\right|_{r=R} .
$$

The first stands for the continuity of the wavefunction at the interface $r=R$ that is automatically satisfied due to the suitable choice of the form of $\phi_{1}$ and $\phi_{2}$. The second represents the continuity of the wave function gradient being modified to cope with the different masses on the two sides of the interface. These boundary conditions lead to the transcendental equation

$$
\tan \left(k_{1} r\right)=\frac{k_{1} R}{1-\frac{m_{1}}{m_{2}}\left(1+k_{2} R\right)},
$$

by solving it, we obtain the lowest subband energy $E_{0}$.

In the presence of the impurity (Eq. (1b)) the exact analytical solution can be obtained for the case of central impurity (Mikhail and El Sayed [32]). The exact analytical solution for the impurity state $(2 p)$ will be given in Sect. 4 in the present article. For the off-center impurity, no exact solution has been inferred. Thus the variational principle is the preferable technique that can be applied in the case of an off-center impurity. This technique will be explored in the following section.

\section{Off-center impurity}

As has been pointed out at the end of the previous section, the case of an off-center impurity can only be dealt with by using the variational principle. We further follow the same approach used by Bella and Navaneethakrishnan [22], Chaudhuri and Bajaj [47], Latge et al. [48] and Villamil et al. [49, 50] and take the part of the variational trial wave function that represents the electron in the QD to be in the ground state (Eq. (5)) for any hydrogenic impurity state.

The trial wave function will thus be taken in the form

$$
\psi_{n l m}(\boldsymbol{r})=N \phi(r) \Gamma_{n l m}(\boldsymbol{r}),
$$

where $\phi(r)$ takes the same form as Eq. (5) but without the normalization constant $N . \Gamma_{n l m}(r)$ is the part of the trial wave function that represents the hydrogenic impurity in the state determined by the quantum numbers $n, l, m$. In the present article the state $(2 p)$ will be considered and accordingly $n=2, l=1, m=0$. The states $\left(2 p_{ \pm}\right)(m= \pm 1)$ will not be considered here as it has been shown in Ref. [22] that these states yield the same results as the state $(2 p)(m=0)$ for central impurity. For off-center impurity, the results should differ. However, this point is postponed to be investigated in a future article.

As regards $\Gamma_{210}$ of the excited state $(2 p)$, two different forms will be considered. The first is the conventional form which has been used in most of the earlier works on quantum dots. The second is a new form that resembles the new forms which have been introduced for the ground state $(1 s)$ in Mikhail and El Sayed [44, 45, 32] for the cases of QWW, coaxial QWW and multilayered QD, respectively. These two forms will be considered in the following two subsections.

\subsection{Conventional wave function}

Here, we follow the same approach used by earlier workers and utilize in the present case of different masses the same trial wave function that has been used in the case of equal masses. We thus take

$$
\Gamma_{210}(r, \theta)=X \cos \theta \mathrm{e}^{-\lambda X},
$$

where

$$
X=\left|\boldsymbol{r}-\boldsymbol{r}_{0}\right|=\left(r^{2}+r_{0}^{2}-2 r r_{0} \cos \theta\right)^{1 / 2},
$$

$\boldsymbol{r}=(r, \theta, \Phi)$ and $\lambda$ is the variational parameter. From now on $\Gamma_{210}$ will be denoted by $\Gamma$ for simplicity. Also, the normalization constant $N$ in Eq. (9) is determined from the normalization condition

$$
\langle\psi(r), \psi(r)\rangle=1 \text {. }
$$

The expectation energy eigenvalue of the operator $\hat{H}_{1}$ is given by

$$
E=\left\langle\psi, \hat{H}_{1} \psi\right\rangle
$$

and the corresponding binding energy of the impurity is consequently given by

$$
E_{\mathrm{b}}=E_{0}-E_{\text {min }} \text {. }
$$

The value of $\lambda$ is obtained by minimizing the energy $E$ in Eq. (12).

In order to derive analytical expressions for $E$, we use the relations

$$
\nabla^{2} \psi=\Gamma \nabla^{2} \phi(r)+2 \nabla \phi \cdot \nabla \Gamma+\phi(r) \nabla^{2} \Gamma
$$
and

$$
\begin{aligned}
& 2\langle\psi, \nabla \phi \cdot \nabla \Gamma\rangle_{i}=W_{i}-\left\langle\psi, \phi \nabla^{2} \Gamma\right\rangle_{i}-\left\langle\phi, \phi|\nabla \Gamma|^{2}\right\rangle_{i}, \\
& \quad i=1,2,
\end{aligned}
$$

where

$$
W_{i}=2 \pi \int_{0}^{\pi}\left(\phi_{i}^{2} \frac{\partial \Gamma}{\partial r} \Gamma r^{2}\right)_{R_{i-1}}^{R_{i}} \sin (\theta) \mathrm{d} \theta, \quad i=1,2,
$$

$R_{0}=0, R_{1}=R, R_{2}=\infty$.

The results in Eqs. (15a, b) have been obtained by integrating by parts. They are consistent with the results given in Eqs. (11a, b) of Ref. [32] for the multilayered QD. It can then be shown after some algebra that $E$ is given by

$$
\begin{aligned}
E & =E_{0}+\left[-8 r_{0}^{2}\left(Q_{1}+\frac{m_{1}}{m_{2}} Q_{2}\right)-\frac{1}{R^{2}}\left(1-\frac{m_{1}}{m_{2}}\right)\right. \\
& \left.\times \phi_{1}^{2}(R) W+4\left(D_{1}+D_{2}\right)\right] / \frac{\mathrm{d}}{\mathrm{d} \lambda}\left(D_{1}+D_{2}\right),
\end{aligned}
$$

where

$$
\begin{aligned}
Q_{i} & =\int_{R_{i-1}}^{R_{i}} \phi_{i}^{2} F r \mathrm{~d} r, \\
D_{i} & =\int_{R_{i-1}}^{R_{i}} \phi_{i}^{2} \frac{J}{r} \mathrm{~d} r, \quad i=1,2, \\
F & =\int_{\left|r-r_{0}\right|}^{r+r_{0}} X|\nabla \Gamma|^{2} \mathrm{~d} X, \\
J & =\int_{\left|r-r_{0}\right|}^{r+r_{0}} X^{2}\left(r^{2}+r_{0}^{2}-X^{2}\right)^{2} \mathrm{e}^{-2 \lambda X} \mathrm{~d} X, \\
W & =\int_{R-r_{0}}^{R+r_{0}} \mathrm{e}^{-2 \lambda X}(\lambda X-1)\left(R^{2}+r_{0}^{2}-X^{2}\right)^{2}
\end{aligned}
$$




$$
\times\left(X^{2}+R^{2}-r_{0}^{2}\right) X \mathrm{~d} X,
$$

and

$$
\begin{aligned}
& |\nabla \Gamma|^{2}=\mathrm{e}^{-2 \lambda X}\left\{\left[\cos \theta(1-\lambda X)+\frac{X \sin \theta}{r}\right]^{2}\right. \\
& \left.-\frac{\sin 2 \theta}{r}(1-\lambda X)\left(X+r_{0} \sin \theta\right)\right\} .
\end{aligned}
$$

In (17f) $|\nabla \Gamma|^{2}$ has to be expressed in terms of $X$ by using Eq. (10).

Now since the spherical harmonics $Y_{l m}(\theta, \phi)$ are included in the form of $\Gamma_{n l m}$ the angular momentum term $\hat{L}^{2}$ in the form of the spin operator $\hat{B}$ can be replaced by $\hbar^{2} l(l+1)$. Also, an electron eigenspinor $\left|\frac{1}{2}, \pm \frac{1}{2}\right\rangle$ has to be added to the form of the trial wavefunction $\psi(r)$ (Eq. (9)) in order to replace the operators $\hat{J}^{2}$ and $\hat{S}^{2}$ in the form of $\hat{B}$ by $\hbar^{2} j(j+1)$ and $3 \hbar^{2} / 4$. Accordingly

$$
\hat{B} \equiv \frac{\hbar^{2}}{4 m^{* 2} c^{2} R^{*} a^{* 3}}[j(j+1)-l(l+1)-3 / 4],
$$

where the factor $1 /\left(R^{*} a^{* 3}\right)$ has been added to adjust the units of the energy and $r$ in the second term of Eq. (1a). Also, $j=l \pm 1 / 2$ and thus $j=\frac{3}{2}, \frac{1}{2}$ for the state $(2 p)$ $(l=1)$. It is readily shown that the expectation energy due to the spin-orbit interaction will take the form

$$
E_{\text {s.o. }}=-2 b \alpha_{j}\left[S_{1}+\left(\frac{m_{1}}{m_{2}}\right)^{2} S_{2}\right] / \frac{\mathrm{d}}{\mathrm{d} \lambda}\left(D_{1}+D_{2}\right),
$$

where

$$
\begin{aligned}
& S_{i}=\int_{R_{i-1}}^{R_{i}} \phi_{i}^{2} M \frac{\mathrm{d} r}{r}, \quad i=1,2, \\
& M=\int_{\left|r-r_{0}\right|}^{\left(r+r_{0}\right)} \mathrm{e}^{-2 \lambda X}\left(r^{2}+r_{0}^{2}-X^{2}\right)^{2} \mathrm{~d} X
\end{aligned}
$$

and

$$
b=\frac{R^{*}}{m_{1} c^{2}}, \quad \alpha_{3 / 2}=1, \quad \alpha_{1 / 2}=-2 .
$$

The eigenspinors $\left|\frac{1}{2}, \pm \frac{1}{2}\right\rangle$ will not affect the previous calculations of the binding energy. The value of $E_{\text {s.o. }}$ calculated from Eq. (19) was found to be much less (less by a factor of the order of $10^{-5}$ ) than the energy $E$ calculated from Eq. (16). We have thus preferred to calculate the variational parameter $\lambda$ from the minimization of the energy $E$ in Eq. (16) prior to the calculation of $E_{\text {s.o. }}$. An alternative approach is to include $E_{\text {s.o. in Eq. (16) }}$ in order to take part in the minimization procedure and in the determination of $\lambda$. However, this approach will obviously give rise to some complications in the energy expression used in the calculation of $\lambda$ without giving any appreciable change in its value.

\subsection{New form of the trial wave function}

The conventional form of the trial wave function Eq. (9) with $\Gamma$ being defined from Eq. (10) does not satisfy the second boundary condition in Eq. (7) in the case of different masses. This, in turn, indicates that the form of $\Gamma$ given in Eq. (10) may not be a suitable choice if different masses are considered. It has to be replaced by a new form so that the resulting trial wave function $\psi(r)$ satisfies the required boundary conditions. Here we introduce a new form of $\Gamma$ for the state $(2 p)$ that is analogous to those used in Mikhail and El Sayed [44, 45, 32] for the state $(1 s)$. Accordingly we take

$$
\Gamma(r, \theta)=\left\{\begin{array}{c}
\Gamma_{1}=X \cos \theta \mathrm{e}^{-\lambda X}, \\
0<r<R, \\
\Gamma_{2}=\frac{X^{\frac{m_{2}}{m_{1}}}}{X_{1}^{\left(\frac{m_{2}}{m_{1}}-1\right)}} \cos \theta \mathrm{e}^{-\lambda\left(1-\frac{m_{2}}{m_{1}}\right) X_{1}} \mathrm{e}^{-\lambda \frac{m_{2}}{m_{1}} X}, \\
R<r<\infty,
\end{array}\right.
$$

where

$$
X_{1}=\left(R^{2}+r_{0}^{2}-2 R r_{0} \cos \theta\right)^{1 / 2} .
$$

It is readily shown that $\psi(r)$ as defined by Eq. (9) with $\Gamma$ given by Eq. (21) satisfies the boundary conditions (Eq. (7)) as is required.

Now, by using the new form of the trial wave function and the same type of calculations used in the previous section, it can be shown that Eq. (15a) still takes the same form but without the term $W_{i}$ on the right hand side (rhs). This term has been obliterated due to the fact that the new trial wave function satisfies the required boundary conditions. This seems to be one of the advantages of the present approach. Finally the expectation value of the operator $\hat{H}_{1}$ is given by

$$
\begin{aligned}
E=E_{0}+\left[\left(\tilde{Q}_{1}+\frac{m_{1}}{m_{2}} \tilde{Q}_{2}\right)-2\left(\tilde{D}_{1}+\tilde{D}_{2}\right)\right] \\
\\
/\left(\tilde{P}_{1}+\tilde{P}_{2}\right),
\end{aligned}
$$

where

$$
\begin{aligned}
\tilde{Q}_{i} & =\int_{0}^{\pi} \int_{R_{i-1}}^{R_{i}} \phi_{i}^{2}(r)\left|\nabla \Gamma_{i}\right|^{2} r^{2} \sin \theta \mathrm{d} r \mathrm{~d} \theta \\
\tilde{D}_{i} & =\int_{0}^{\pi} \int_{R_{i-1}}^{R_{i}} \phi_{i}^{2}(r) \Gamma_{i}^{2}(r, \theta) \frac{r^{2} \sin \theta}{X} \mathrm{~d} r \mathrm{~d} \theta \\
\tilde{P}_{i} & =\int_{0}^{\pi} \int_{R_{i-1}}^{R_{i}} \phi_{i}^{2}(r) \Gamma_{i}^{2}(r, \theta) r^{2} \sin \theta \mathrm{d} r \mathrm{~d} \theta, \quad i=1,2, \\
\mid \nabla & \left.\Gamma_{2}\right|^{2}=\left(\frac{m_{2}}{m_{1}}\right)^{2} \Gamma_{2}^{2}\left[\left(\frac{1}{X}-\lambda\right)^{2}+2\left(\frac{1}{X}-\lambda\right)\right. \\
& \left.\times \frac{r_{0} \sin \theta}{X} U+U^{2}\right], \\
U & =\left(\frac{m_{1}}{m_{2}}-1\right)\left(\frac{1}{X_{1}}-\lambda\right) \frac{r_{0} R \sin \theta}{X_{1} r}-\frac{m_{1}}{m_{2}} \frac{\tan \theta}{r},
\end{aligned}
$$

and $\left|\nabla \Gamma_{1}\right|^{2}$ is defined as in Eq. (17f).

Also $E_{\text {s.o. }}$ is given by

$$
E_{\text {s.o. }}=b \alpha_{j}\left[\tilde{S}_{1}+\left(\frac{m_{1}}{m_{2}}\right)^{2} \tilde{S}_{2}\right] /\left(\tilde{P}_{1}+\tilde{P}_{2}\right),
$$

where

$$
\tilde{S}_{i}=\int_{0}^{\pi} \int_{R_{i-1}}^{R_{i}} \phi_{i}^{2}(r) \Gamma_{i}^{2}(r, \theta) \frac{r^{2} \sin \theta}{X^{3}} \mathrm{~d} r \mathrm{~d} \theta, \quad i=1,2 .
$$

The results in the two special cases of equal masses and of central impurity can be obtained by taking $m_{1}=m_{2}$ 
and by taking the limit when $r_{0} \rightarrow 0$, respectively, in the expressions obtained for $E, E_{\text {s.o. }}$ in Sects. 3.1, 3.2. They are not given here due to their length and since the derivations are straightforward.

As was pointed out previously an exact analytical solution for the Schrödinger equation corresponding to the Hamiltonian $\hat{H}_{1}$ in the case of central impurity and different masses has been derived in Mikhail and El Sayed [32]. However, the method has only been applied in [32] for the ground state $(1 s)$. The solution in the excited state $(2 p)$ will be presented in the following section together with the corresponding calculations of the spin-orbit interaction term.

\section{Exact analytical solution - for central impurity}

It is readily shown that the radial Schrödinger equation corresponding to $\hat{H}_{1}$ in the presence of a central impurity $\left(r_{0}=\mathbf{0}\right)$ can be put in the form

$$
\frac{1}{\psi(\rho)} \frac{\mathrm{d}}{\mathrm{d} \rho} \rho^{2} \frac{\mathrm{d} \psi}{\mathrm{d} \rho}-l(l+1)+\rho^{2}\left(-\frac{1}{4}+\frac{\lambda}{\rho}\right)=0,
$$

where $\rho_{i}=\gamma_{i} r(i=1,2), \gamma_{1}=2 \sqrt{-E}, \lambda_{1}=\frac{2}{\gamma_{1}}=\frac{1}{\sqrt{-E}}$, and

$$
\begin{aligned}
\gamma_{2} & =2 \sqrt{\frac{m_{2}}{m_{1}}\left(V_{0}-E\right)}, \\
\lambda_{2} & =\frac{2 m_{2}}{m_{1}} \frac{1}{\gamma_{2}}=\sqrt{\frac{m_{2}}{m_{1}\left(V_{0}-E\right)}} .
\end{aligned}
$$

In Eqs. (28) $i=1,2$ represent, respectively, the two regions inside $(0<r<R)$ and outside $(R<r<\infty)$ the dot. It has been consequently shown in Mikhail and El Sayed [32] that the solution of Eq. (27) can be expressed as

$$
\psi(\rho)=\mathrm{e}^{-\rho / 2} \rho^{l} G(\rho),
$$

where $G(\rho)$ is found to be the confluent hypergeometric function ${ }_{1} F_{1}(l+1-\lambda, 2 l+2, \rho)$ or $U(l+1-\lambda, 2 l+2, \rho)$. Thus for the excited state $(2 p)(l=1)$, the exact solutions are given by

$$
\psi(r)=\left\{\begin{array}{l}
\psi_{1}(r)=N \mathrm{e}^{-\rho_{1} / 2} \rho_{1}{ }_{1} F_{1}\left(2-\lambda_{1}, 4, \rho_{1}\right), \\
0<r<R, \\
\psi_{2}(r)=N N_{2} \mathrm{e}^{-\rho_{2} / 2} \rho_{2} U\left(2-\lambda_{2}, 4, \rho_{2}\right), \\
\quad R<r<\infty .
\end{array}\right.
$$

The energy $E$ of the system and the coefficient $N_{2}$ can be determined from the boundary conditions (7) after replacing $\phi(r)$ by $\psi(r)$ while the coefficient $N$ can be obtained from the normalization condition (11). The impurity binding energy is given by

$$
E_{\mathrm{b}}=E_{0}-E \text {. }
$$

Finally, the spin-orbit interaction energy is given by

$$
E_{\text {s.o. }}=b \alpha_{j}\left[\tilde{\tilde{S}}_{1}+\left(\frac{m_{1}}{m_{2}}\right)^{2} \tilde{\tilde{S}}_{2}\right] /\left(\tilde{\tilde{P}}_{1}+\tilde{\tilde{P}}_{2}\right),
$$

where

$$
\tilde{\tilde{S}}_{i}=\int_{R_{i-1}}^{R_{i}}\left|\psi_{i}(r)\right|^{2} \frac{\mathrm{d} r}{r}
$$

$$
\tilde{\tilde{P}}_{i}=\int_{R_{i-1}}^{R_{i}}\left|\psi_{i}(r)\right|^{2} r^{2} \mathrm{~d} r, \quad i=1,2 .
$$

\section{Numerical results}

The results obtained in the previous sections are applied in the present section to calculate the spin-orbit interaction energy in the case of a central and off-central impurity located in a $\mathrm{GaAs}-\mathrm{Ga}_{1-x} \mathrm{Al}_{x} \mathrm{As}$, spherical quantum dot. The required input parameters are the confining potential $V_{0}$, the effective masses $m_{1}, m_{2}$ and the dielectric constant $\epsilon_{\mathrm{r}}$. They are determined in terms of the Al concentration $x$ by the following relations according to Casey [51] and Adachi [52]:

$$
\begin{aligned}
& V_{0}=0.7482 x(\mathrm{eV}), \quad \epsilon_{\mathrm{r}}=12.58, \\
& m_{1}=0.067 m_{0}, \quad m_{2}=(0.067+0.083 x) m_{0} .
\end{aligned}
$$

In the following calculations $V_{0}=147.4 \mathrm{meV}$ which is the same value used in Ref. [22]. It corresponds to $x=0.197$. Also, the values of the effective Rydberg $R^{*}$ and effective Bohr radius $a^{*}$ are given by

$$
R^{*} \approx 5.72 \mathrm{meV} \text { and } a^{*} \approx 100 \AA \text {. }
$$

The energy $E_{0}$ in the absence of impurity and spin-orbit interaction is determined from Eqs. (6), (8). Moreover, the following results are only given for $j=\frac{3}{2}$. The results for $j=\frac{1}{2}$ can be easily obtained by multiplying the results for $j=\frac{3}{2}$ by a factor $(-2)$.

In Fig. 1, the spin-orbit energy is displayed as a function of the dot radius in the case of a central impurity. A comparison is given between the results obtained by using the exact solution (Eq. (32)) (case 1) and by using the variational method with the new trial wave function (Eqs. (26a, b)) (case 2) and with the conventional trial wave function (Eqs. (19), (20)) (case 3). Different factors have influenced the choice of the minimum value of $R$ in this figure. According to Mikhail and Ismail [39] in the absence of impurity

$$
\left(R_{\min }\right)_{1 s}=\pi /\left(2 \sqrt{V_{0}}\right) \approx 0.31 a^{*}
$$

and

$$
\left(R_{\min }\right)_{2 p}=\pi / \sqrt{V_{0}} \approx 0.62 a^{*} .
$$

In spite of the fact that in the absence of impurity the electron is taken to be in the state $(1 s)$ (Eq. (5)), it is found that in the presence of the central impurity in the state $(2 p)$ the expectation energy (determined from Eq. (16) or Eq. (23)) approaches $V_{0}$ as $R \rightarrow 0.62 a^{*}$. Thus, for the variational method we have restricted the calculations to begin from $R=0.634 a^{*}$ if the conventional form of the trial wave function is used and from $R=0.556 a^{*}$ if the new form of the trial wave function is used. On the other hand, for the exact solution, no minimum critical dot radius exists since the potential of the impurity is not a square well potential but is proportional to $1 / r$ (Yang et al. [21]). The results can thus be extended to a very small dot radius $\left(R=0.1 a^{*}\right)$.

The general behavior of the three sets of results shown in Fig. 1 is similar. The spin-orbit energy increases as the dot radius decreases until it reaches a maximum value (cases 1 and 2) about $R=0.6 a^{*}$. It starts to decrease for

and 


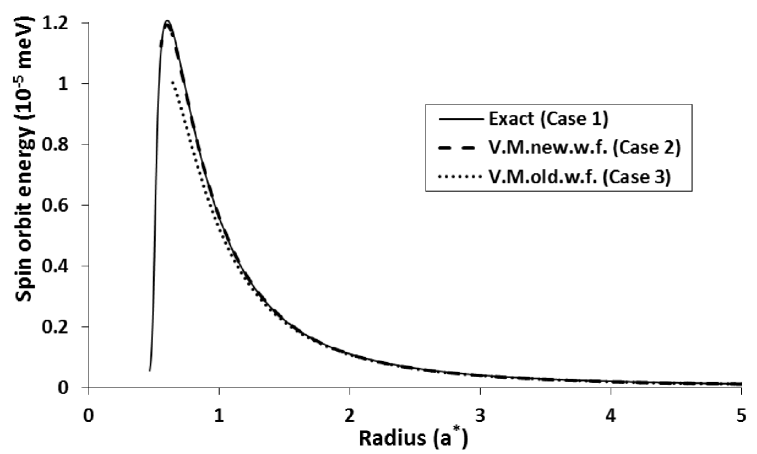

Fig. 1. Spin-orbit energy ( $\left.E_{\text {s.o. }}\right)$ against the dot radius $(R)$ for a central impurity.

$R<0.6 a^{*}$ since the probability of the electron tunneling to the barrier increases for dots of small radius.

The results obtained by using the exact solution (case 1) are higher than the other two cases. It is higher by about $8.59 \%$ and $14.6 \%$ at $R=5 a^{*}$ and $R=0.64 a^{*}$, respectively, than the results obtained by using the conventional trial wavefunction while it is higher by about $8.54 \%$ and $1.36 \%$ at $R=5 a^{*}$ and $R=0.64 a^{*}$ than the results obtained by using the new trial wave function. It is thus clear that the results obtained by using the new form of the trial wave function approach the exact solution at small values of $R$.

It is also of importance to point out that the present results are less by an order of magnitude than the results of Refs. [22, 23]. This seems to be due to the numerical value of the factor $(b)$ in Eqs. (19), (20c). In the present work we have found that

$$
b=0.095432 \times 10^{-5} \mathrm{meV}=0.016684 \times 10^{-5} R^{*} .
$$

The corresponding value in Refs. [22, 23] has been estimated and was found to be

$$
b=1.2059 \times 10^{-5} \mathrm{meV} \text {. }
$$

Thus

$$
(b)_{\text {Eq. }(35 b)} /(b)_{\text {Eq. }(35 a)} \approx 12.636,
$$

which is equivalent to $(4 \pi)$ or to $\left(\epsilon_{\mathrm{r}}\right)$. We have calculated the value of $(b)$ in Eq. (35a) in several ways and to the best of our knowledge it seems to be correct. Thus either the factor $(4 \pi)$ or $\left(\epsilon_{\mathrm{r}}\right)$ is missing in the results of Refs. $[22,23]$.

Also, in order to compare the present results with the results reported in Yang et al. [21] and Özmen et al. [25], we have used the input parameters

$$
\begin{aligned}
& m_{1}=m_{2}=m_{0}, \quad \epsilon_{\mathrm{r}}=1, \quad V_{0}=5 \mathrm{eV}, \\
& R^{*}=13.6 \mathrm{eV}, \quad a^{*}=0.529 \AA,
\end{aligned}
$$

of the free space hydrogen atom. This gives

$$
b=0.361455 \mathrm{meV}=0.0265776 \times 10^{-3} R^{*} .
$$

The results obtained for the spin-orbit interaction in the case of central impurity using the above input parameters together with the corresponding results of Yang et al. [21] are given in Table. The above comparison shows that the results obtained using the exact solution are identi- cal with the results of Yang et al. [21]. The difference between the two sets is less than $0.4 \%$. The results obtained by using the variational method are lower than the results of the exact solution and than the results of Yang et al. [21] by about $12 \%$. Here the two forms of trial wave function (new and conventional) are equivalent since $m_{1}=m_{2}$. Also, the results of the spin-orbit interaction of Refs. [21] and [25] are consistent. For this

\begin{tabular}{|c|c|c|c|}
\hline \multirow{2}{*}{$R\left(a^{*}\right)$} & \multirow{2}{*}{$\begin{array}{c}E_{\text {s.o. }}\left(10^{-2} \mathrm{meV}\right) \\
\text { Yang et al. }[21]\end{array}$} & \multicolumn{2}{|c|}{$\begin{array}{c}E_{\text {s.o. }}\left(10^{-2} \mathrm{meV}\right) \\
\text { Present results }\end{array}$} \\
\hline & & $\begin{array}{l}\text { Variational } \\
\text { method }\end{array}$ & $\begin{array}{l}\text { Exact } \\
\text { solution }\end{array}$ \\
\hline 0.1 & 1.51 & - & 1.5061 \\
\hline 4 & 3.67 & 3.4036 & 3.655 \\
\hline 8 & 1.9 & 1.6586 & 1.8922 \\
\hline 17 & 1.51 & 1.4316 & 1.508 \\
\hline
\end{tabular}
reason, the comparison in Table is given only between the present results and those of Ref. [21].

TABLE

Comparison between the present results of spin-orbit interaction and the results of Yang et al. [21].

Regarding the recent published article of Yakar et al. [24], they have calculated the results in the case of infinite confining potential QD with the same input parameters of GaAs given in Eqs. (34a,b,c) of the present work $\left(x=0, m_{1}=m_{2}=0.067 m_{0}\right)$. They, however, stated that their results of $E_{\text {s.o. }}$ tends to the value of hydrogen atom for quantum dots of large radius in atomic units. This contradicts the fact that the parameter $(b)$

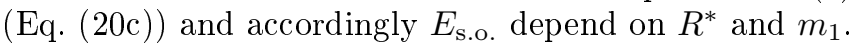
It thus differs in atomic units from one set of input parameters to another, unlike the electron energy, Coulomb interaction and binding energy. It seems, therefore, that the results of Yakar et al. [24] are incorrect if the input parameters of GaAs are used. In order to confirm this conclusion we have calculated the value of $E_{\text {s.o. }}$ in the case of infinite confining potential with the GaAs input parameters (Eq. (34a, second part) and Eqs. (34b,c, with $x=0)$ ). The results obtained in atomic units are less than those of Yakar et al. [24] by an average factor 159 . In fact we have found that this factor is equivalent to the ratio between the value of the parameter $(b)$ of the free space hydrogen atom in atomic units (Eq. (37)) and the parameter (b) of GaAs in atomic units (Eq. (35a)). This again indicates that in Yakar et al. [24] the spin-orbit interaction has been calculated for a free space hydrogen atom unlike what has been stated that the calculations have been performed for an impurity located in a GaAs quantum dot.

The rest of the calculations in this section are performed using the input parameters given in Eqs. (34a,b,c) only. In order to clarify the effect of using the different masses of dot and barrier we give in Fig. 2 a comparison between the results of spin-orbit interaction obtained by using the exact solution with different (case 1) and equal (case 2) masses. The results in the case of equal masses 


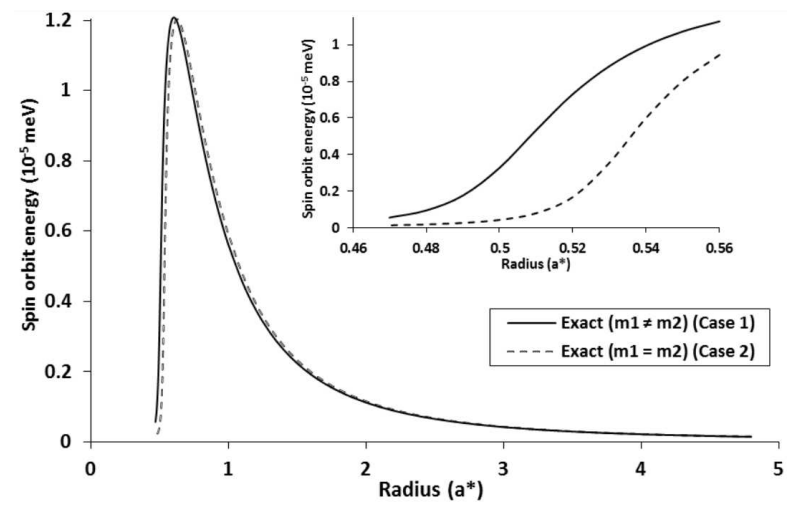

Fig. 2. Comparison between the spin-orbit energy for a central impurity obtained using the exact solution in the cases of different (case 1) and equal (case 2) masses. The inset shows the results in the range $0.47 a^{*}<R<$ $0.56 a^{*}$.

(case 2) are higher than the results of different masses (case 1) for $R>0.63 a^{*}$ in spite of the fact that the binding energies in case 2 are less than the corresponding binding energies in case 1 . This may be attributed to the factor $\left(m_{1} / m_{2}\right)^{2}$ that appears in the second term of the numerator in Eq. (32). The results of equal masses (case 2 ) are higher by about $1 \%$ and $1.56 \%$ at $R=5 a^{*}$ and $R=0.64 a^{*}$, respectively, than the results of different masses (case 1). However, for $R<0.63 a^{*}$, the results in case 1 starts to be higher than the results in case 2 . This is due to the fact that the consideration of different masses decreases the probability of tunneling at small $R$. The difference between the two sets of results becomes of the order of $25 \%$ at $R=0.55 a^{*}$ and of the order of $86.5 \%$ at $R=0.5 a^{*}$. This confirms that the consideration of the different masses (case 1) has a strong influence on the spin-orbit energy in this range. The results displayed in the inset of Fig. 2 emphasize further this conclusion.

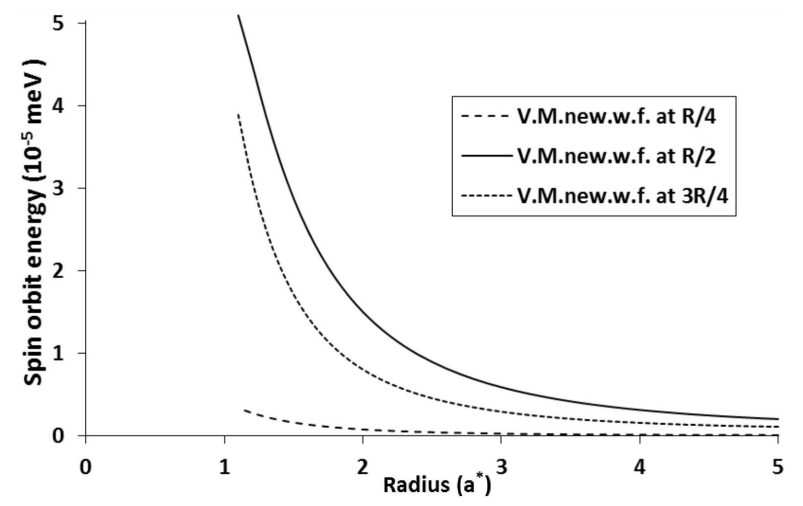

Fig. 3. Plot of spin-orbit energy ( $\left.E_{\text {s.o. }}\right)$ of an off-central impurity against the dot radius $(R)$. The variational method is used with the new trial wavefunction.

The case of off-center impurity is shown in Fig. 3 for $r_{0}=R / 4, R / 2,3 R / 4$. In this case no exact analytical solution has been obtained. Thus the results presented in Fig. 3 are obtained by using the new form of the trial wave function for which the spin-orbit energy approaches the exact solution for a central impurity (Fig. 1). For all the impurity locations the spin-orbit energy increases as the dot radius decreases within the range of $R$ considered. Also the results obtained for $r_{0}=R / 2$ are higher than those of $r_{0}=R / 4$ and $r_{0}=3 R / 4$. This clarifies the fact that the spin-orbit interaction increases as $r_{0}$ increases until it becomes maximum near the position at which the probability of finding an electron in the state $(2 p)$ is maximum. The same behavior was also exhibited for the binding energy $E_{\mathrm{b}}$ in Mikhail and Ismail paper [39].

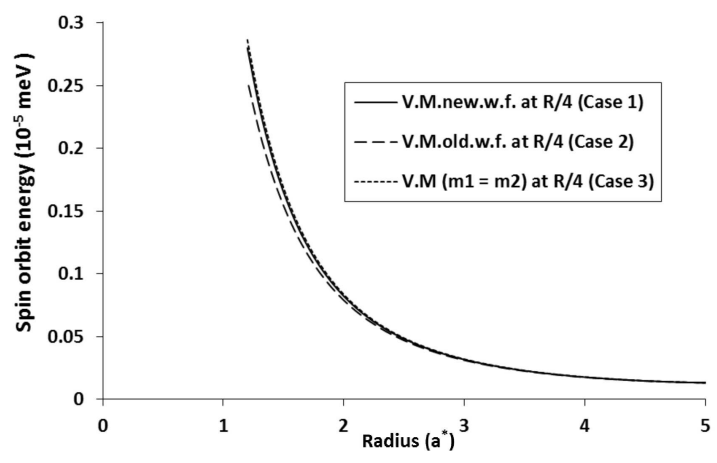

Fig. 4. Comparison between the spin-orbit energy for an off-central impurity $\left(r_{0}=R / 4\right)$ for the cases of different masses $\left(m_{1} \neq m_{2}\right)$ and equal masses $\left(m_{1}=m_{2}\right)$.

In Fig. 4 the spin-orbit energy is given for an off-central impurity located at $r_{0}=R / 4$ in the cases of different masses $\left(m_{1} \neq m_{2}\right)$ using the new (case 1) and conventional (case 2) wave functions and of equal masses $\left(m_{1}=m_{2}\right)$ (case 3$)$. The comparison shows that the results obtained in case 1 (new wave function) are higher than those obtained in case 2 (conventional wave function) by about $5.28 \%$ at $R=1.7 a^{*}$ and by about $0.33 \%$ at $R=5 a^{*}$. Moreover, the results obtained for equal masses (case 3 ) are higher by about $1.75 \%$ and $2.69 \%$ at $R=5 a^{*}$ from the results of cases 1 and 2 , respectively. Also, they are higher by about $1.94 \%$ and $7.63 \%$ at $R=1.7 a^{*}$ from the results of cases 1 and 2 . Thus the results of an off-central impurity confirm the same conclusion found in the case of central impurity (Fig. 2) that the consideration of the different masses of dot and barrier decreases the spin-orbit energy for $R>0.63 a^{*}$.

\section{Conclusions}

In all previous treatments the conventional spin-orbit interaction has been calculated for a central impurity assuming equal masses of dot and barrier. In the present work we have performed the calculations for both off-central and central impurities considering different masses of dot and barrier. The different masses have to be taken into consideration in the case of GaAs$\mathrm{Ga}_{1-x} \mathrm{Al}_{x}$ As finite confining potential dot due to the dependence of the barrier effective mass on the $\mathrm{Al}$ concentration $(x)$. 
The variational method has been applied in the case of off-central and central impurities using two forms of the trial wave function, the conventional form and a new form that satisfies the required boundary conditions in the case of different masses. The new form has considerably improved the results of the spin-orbit interaction.

In the case of central impurity the spin-orbit interaction has been also calculated using the exact solution of the radial SChrödinger equation in the presence of the impurity for the state $(2 p)$. The results are higher than those obtained using the variational method. However, the results of the new form of the trial wave function approach those obtained using the exact solution for dots of small radius. Also, the results obtained using the exact solution indicate that the consideration of the different masses has a significant effect on the spin-orbit energy for $R<0.63 a^{*}$.

The results have been compared with the results reported in earlier work. The results obtained using the exact solution for a central impurity are identical with the results of Yang et al. [21] and of Özmen et al. [25] to within $0.4 \%$. Regarding the other earlier treatments we have suggested some amendments to reconcile their results with the present results. In this connection, it is worthwhile pointing out that the comparison has been made only for central impurity since all earlier treatments were performed in this case. Moreover some of the methods used in these treatments (Yang et al. [21], Yakar et al. [24] and Özmen et al. [25]) cannot be applied for off-central impurities.

Finally, we would like to confirm that in spite of the small order of magnitude of spin-orbit interaction with respect to the electron energy and binding energy it can be separately measured by spintronic devices where it plays the essential role.

\section{References}

[1] O. Voskoboynikov, C.P. Lee, O. Tretyak, Physica E 10, 107 (2001).

[2] X.F. Wang, P. Vasilopoulos, Phys. Rev. B 68, 035305 (2003).

[3] X.F. Wang, Phys. Rev. B 69, 035302 (2004).

[4] M. Florescu, S. Dickman, M. Ciorga, A. Sachrajda, P. Hawrylak, Physica E 22, 414 (2004).

[5] K. Gnanasekar, K. Navaneethakrishnan, Physica E 35, 103 (2006).

[6] P. Kleinert, Physica E 41, 660 (2009).

[7] G. Gumbs, D. Huang, Phys. Lett. A 373, 2506 (2009).

[8] T.F.A. Alves, A.C.A. Ramos, G.A. Farias, R.N.C. Filho, N.S. Almeida, Eur. Phys. J. B 67, 213 (2009).

[9] C.L. Romano, P.I. Tamborenea, S.E. Ulloa, Physica E 41, 1577 (2009).

[10] C. Bai, J. Wang, J. Tian, Y. Yang, Physica E 43 , 207 (2010).

[11] E. Papp, C. Micu, Superlatt. Microstruct. 48, 9 (2010)
[12] Y.C. Xiao, W.J. Deng, Superlatt. Microstruct. 48, 181 (2010).

[13] A. Vanitha, C.W. Lee, A.J. Peter, Phys. Lett. A 375 , 208 (2010).

[14] P.M. Krstajic, M. Pagano, P. Vasilopoulos, Physica E 43, 893 (2011)

[15] H. Hassanabadi, H. Rohimov, S. Zarrinkamar, Ann. Phys. 326, 2957 (2011)

[16] M.P. Nowak, B. Szafran, F.M. Peeters, B. Partoens, W. Pasek, arXiv:1102. 1002v2 [cond-mat. mes-hall], (2011), see xxx.lanl.gov .

[17] H. Hassanabadi, H. Rohimov, L. Lu, C. Wang, J. Lumin. 132, 1095 (2012).

[18] S.K. Maiti, S. Sil, A. Chakrabarti, Phys. Lett. A 376, 2147 (2012).

[19] J.D. Lu, Y.B. Li, Superlatt. Microstruct. 48, 517 (2010).

[20] B. Veseghi, G. Rezaei, V. Azizi, F. Taghizadeh, Physica E 43, 1080 (2011).

[21] C.C. Yang, L.C. Liu, S.H. Chang, Phys. Rev. B 58 1954 (1998).

[22] R.S.D. Bella, K. Navaneethakrishnan, Solid State Commun. 130, 773 (2004).

[23] S. Rajashabala, K. Navaneethakrishnan, Physica E 40, 843 (2008).

[24] Y. Yakar, B. Çakir, A. Özmen, J. Lumin. 134, 778 (2013).

[25] A. Özmen, B. Çakir, Y. Yakar, J. Lumin. 137, 259 (2013).

[26] J.L. Zhu, Phys. Rev. B 39, 8780 (1989).

[27] J.L. Zhu, J.J. Xiong, B.L. Gu, Phys. Rev. B 41, 6001 (1990).

[28] J.L. Zhu, Z.Q. Li, J.Z. Yu, K. Ohno, Y. Kawazoe, Phys. Rev. B 55, 15819 (1997).

[29] J.L. Zhu, X. Chen, Phys. Rev. B 50, 4497 (1994).

[30] D.S. Chuu, C.M. Hsiao, W.N. Mei, Phys. Rev. B 46 , 3898 (1992).

[31] S. Goldman, C. Joslin, J. Phys. Chem. 96, 6021 (1992).

[32] I.F.I. Mikhail, S.B.A. El Sayed, Physica E 43, 1371 (2011).

[33] N.P. Montenegro, S.T.P. Merchancano, Phys. Rev. B 46, 9780 (1992).

[34] A.J. Peter, Physica E 28, 225 (2005).

[35] C. Dane, H. Akbas, S. Minez, A. Guleroglu, Physica E 41, 278 (2008).

[36] C. Dane, H. Akbas, S. Minez, A. Guleroglu, Physica E 42, 1901 (2010).

[37] H. Akbas, C. Dane, A. Guleroglu, S. Minez, Physica E 41, 605 (2009).

[38] I.F.I. Mikhail, I.M.M. Ismail, Phys. Status Solidi B 244, 3647 (2007).

[39] I.F.I. Mikhail, I.M.M. Ismail, Superlatt. Microstruct. 48, 388 (2010).

[40] B. Çakir, A. Özmen, Ü. Atav, H. Yüksel, Y. Yakar, Int. J. Mod. Phys. C 19, 599 (2008).

[41] A. Özmen, Y. Yakar, B. Çakir, Ü. Atav, Opt. Commun. 282, 3999 (2009). 
[42] Y. Yakar, B. Çakir, A. Özmen, Opt. Commun. 283, 1795 (2010).

[43] Y. Yakar, B. Çakir, A. Özmen, Commun. Theor. Phys. 53, 1185 (2010).

[44] I.F.I. Mikhail, S.B.A. El Sayed, Int. J. Mod. Phys. B 23, 2107 (2009).

[45] I.F.I. Mikhail, S.B.A. El Sayed, Physica E 42, 2307 (2010).

[46] D.J. Griffiths, Introduction to Quantum Mechanics, Pearson Prentice Hall, New Jersey 2005.

[47] S. Chaudhuri, K.K. Bajaj, Phys. Rev. B 29, 1803 (1984).
[48] A. Latge, N.P. Montenegro, L.E. Oliveira, Phys. Rev. B 45, 9420 (1992).

[49] P. Villamil, N.P. Montenegro, J.C. Granada, Phys. Rev. B 59, 1605 (1999).

[50] P. Villamil, C. Cabra, N.P. Montenegro, J. Phys., Condens. Matter 17, 5049 (2005).

[51] H.C. Casey, J. Appl. Phys. 49, 3684 (1978).

[52] S. Adachi, J. Appl. Phys. 58, R1 (1985). 